\title{
Counterexample to the 1-Summability of a Divergent Formal Solution to Some Linear Partial Differential Equations
}

\author{
By
}

\author{
Kenji Kurogi
}

(Hiroshima University, Japan)

\begin{abstract}
In 2013, Tahara-Yamazawa showed multisummability of formal solutions of some linear partial differential equation under a certain condition on the Newton polygon. In this paper, we give an example such that a divergent formal power series solution is not multisummable when the condition on the Newton polygon by TaharaYamazawa does not hold.

Key Words and Phrases. 1-Summability, Partial differential equation, Newton polygon.

2010 Mathematics Subject Classification Numbers. 35C10, 35A20.
\end{abstract}

\section{Motivation and the result}

Let $(t, x)=\left(t, x_{1}, x_{2}, \ldots, x_{N}\right) \in \boldsymbol{C} \times \boldsymbol{C}^{N}$. For $r>0$ we write $D_{r}=\{t \in \boldsymbol{C} \mid$ $|t|<r\}$. For an open set $W$ we denote by $\mathcal{O}(W)$ the set of all holomorphic functions on $W$. For a function $g(t) \in \mathcal{O}(W)$ we denote by $\operatorname{ord}_{t}(g)$ the order of the zero of $g(t)$ at $t=0$.

In this paper, we shall study the multisummability of formal solutions of a linear partial differential equation. This theory has been studied by many mathematicians. Lutz-Miyake-Schäfke [3] studied the Borel summability of divergent solutions of the heat equation. Ouchi [6], [7] studied the multisummability of formal solutions of partial differential equations. For other extensions to partial differential equations, we also refer Malek [4] and Hibino [2]. In [8], Tahara-Yamazawa considered

$$
\left\{\begin{array}{l}
\partial_{t}^{m} u+\sum_{(j, \alpha) \in \Lambda} a_{j, \alpha}(t) \partial_{t}^{j} \partial_{x}^{\alpha} u=f(t, x) \\
\left.\partial_{t}^{j} u\right|_{t=0}=\varphi_{j}(x) \quad(j=0,1, \ldots, m-1),
\end{array}\right.
$$

where $m \geq 1$ is an integer and $\Lambda$ is a finite subset of $\boldsymbol{N} \times \boldsymbol{N}^{N}(\boldsymbol{N}=\{0,1$, $2, \ldots\})$. Here $a_{j, \alpha}(t) \in \mathcal{O}\left(D_{r}\right)((j, \alpha) \in \Lambda), f(t, x) \in \mathcal{O}\left(D_{r} \times C^{N}\right), \varphi_{j}(x) \in \mathcal{O}\left(C^{N}\right)$ $(j=0,1, \ldots, m-1), \partial_{t}=\partial / \partial t$ and $\partial_{x}^{\alpha}=(\partial / \partial x)^{\alpha}=\left(\partial / \partial x_{1}\right)^{\alpha_{1}} \ldots\left(\partial / \partial x_{N}\right)^{\alpha_{N}}, \alpha=$ $\left(\alpha_{1}, \alpha_{2}, \ldots, \alpha_{N}\right) \in N^{N}$. 
For $(a, b) \in \boldsymbol{R}^{2}$ we write $C(a, b)=\{(x, y) \mid x \leq a, y \geq b\}$. The Newton polygon $N_{t}(E)$ corresponding to $(E)$ is defined by

$$
N_{t}(E):=\text { the convex hull of }\left\{C(m,-m) \cup \bigcup_{(j, \alpha) \in A} C\left(j, \operatorname{ord}_{t}\left(a_{j, \alpha}\right)-j\right)\right\} \text {. }
$$

The vertices of $N_{t}(E)$ consist of $p^{*}+1$ points

$$
\left(l_{0}, e_{0}\right),\left(l_{1}, e_{1}\right), \ldots,\left(l_{p^{*}-1}, e_{p^{*}-1}\right),\left(l_{p^{*}}, e_{p^{*}}\right)
$$

and the boundary of $N_{t}(E)$ consists of a horizontal half line $\Gamma_{0}, p^{*}$-segments $\Gamma_{1}, \Gamma_{2}, \ldots, \Gamma_{p^{*}}$, and a vertical half line $\Gamma_{p^{*}+1}$.

In [8], it was assumed that the equation in $(E)$ is Fuchsian and it satisfies the condition

(A1) $\left(j, \operatorname{ord}_{t}\left(a_{j, \alpha}\right)-j\right) \in \bigcup_{i=1}^{p^{*}+1} \Gamma_{i} \Rightarrow|\alpha|=0$, for every $j=0,1, \ldots, m-1$, and that $\varphi_{j}(x)$ and $f(t, x)$ have some exponential size depending on $N_{t}(E)$. Then, it was proved that the formal solution of $(E)$ is multisummable in suitable directions.

We shall study the necessity of (A1). More precisely, we shall give the example which does not satisfy (A1) and whose formal solution is not 1-summable.

Let $(t, x) \in \boldsymbol{C} \times \boldsymbol{C}$. Consider the initial value problem

$$
\left\{\begin{array}{l}
\frac{\partial}{\partial t} u_{1}=a(x)+\left(t \frac{\partial}{\partial t}\right)^{2} u_{1}+\left(t \frac{\partial}{\partial t}\right)^{2} \partial_{x}^{\alpha} u_{1} \\
u_{1}(0, x)=\varphi_{0}(x)
\end{array}\right.
$$

where $\alpha \in N^{*}\left(N^{*}=\{1,2,3, \ldots\}\right), u_{1}=u_{1}(t, x), a(x)$ is an entire function and $\varphi_{0}(x)$ is a holomorphic function on $\boldsymbol{C}$. By taking $u:=u_{1}-\varphi_{0}(x)$ as a new unknown function, $\left(E_{0}\right)$ is equivalent to

$$
t \frac{\partial}{\partial t} u=a(x) t+t\left(t \frac{\partial}{\partial t}\right)^{2} u+t\left(t \frac{\partial}{\partial t}\right)^{2} \partial_{x}^{\alpha} u
$$

with the unknown function

$$
u(t, x)=\sum_{j=1}^{\infty} u_{j}(x) t^{j}
$$

where $u_{j}(x)$ is analytic on $D_{r}$ and continuous up to the boundary for any $j \in \boldsymbol{N}^{*}$. 
Let $n \in N^{*}$ and $q \in N^{*}$ satisfy $q(q-1) / 2<n \leq q(q+1) / 2$. Set $v=$ $n-q(q-1) / 2$ and define $h_{n} \equiv h(n, q)$ by

$$
\frac{1}{1+h_{n}^{\alpha}}=\left(1-\frac{1}{q+1}\right) e^{2 \pi i v /(q+1)},
$$

where $\alpha$ is given in $\left(E_{0}\right)$.

Let $C_{n}$ satisfy $\sum_{n=1}^{\infty}\left|C_{n}\right|<\infty$. Assume that $a(x)$ in (1.1) be given by

$$
a(x)=\sum_{n=1}^{\infty} C_{n} e^{h_{n} x} .
$$

Then we have

Lemma 1.1. Let $h_{n}$ be given by (1.3). Then we have

$$
\left|\frac{1}{1+h_{n}^{\alpha}}\right|=1-\frac{1}{q+1}
$$

and

$$
\arg \left(\frac{1}{1+h_{n}^{\alpha}}\right)=2 \pi \frac{v}{q+1},
$$

for $v=n-q(q-1) / 2, q(q-1) / 2<n \leq q(q+1) / 2$. The function a(x) in (1.4) is well defined.

Proof. (1.5) and (1.6) are immediate consequences of the definition. By (1.5) we have $1 / 2 \leq\left|1 /\left(1+h_{n}^{\alpha}\right)\right|<1$. Hence, we have $\left|h_{n}\right| \leq 3^{\alpha^{-1}}$, and

$$
|a(x)| \leq \sum_{n=1}^{\infty}\left|C_{n}\right|\left|e^{h_{n} x}\right| \leq \sum_{n=1}^{\infty}\left|C_{n}\right| \exp \left(3^{\alpha^{-1}}|x|\right) .
$$

Hence, $a(x)$ converges in $|x|<R$ for every $R>0$.

Then we have the main result of this paper.

Theorem 1.1. Assume (1.4). Then every formal solution (1.2) of (1.1) is not 1-summable in any direction.

Remarks. (a) For $(E)$, Tahara-Yamazawa [8] assumed three conditions, $\left(A_{1}\right),\left(A_{2}\right)$ and $\left(A_{3}\right)$ for some $\gamma \in \mathscr{C}$. See [8] as to the definition of $\mathscr{C}$. $\left(E_{0}\right)$ satisfies $\left(A_{1}\right)$ and does not satisfy $\left(A_{2}\right)$. $\left(E_{0}\right)$ verifies $\left(A_{3}\right)$ with $\gamma \geq 1$, while one sees $\mathscr{C}=(0.1)$ for $\left(E_{0}\right)$. Hence the condition as to $\left(A_{3}\right)$ in [8] does not hold.

(b) Applying Ōuchi's result [5] to $\left(E_{0}\right)$, we see that there is a solution of $\left(E_{0}\right)$ with Gevrey asymptotic expansion (1.2) in a narrow sector. 
In Proposition 3.1 which follows we shall show that the formal solution (1.2) has Gevrey order 1.

This paper is organized as follows. In Section 2, we give some definitions and symbols. In Section 3, we prove Gevrey estimate of the formal solution. The proof of Theorem 1.1 is given in Section 4.

\section{Definitions and notation}

We give some definitions and introduce symbols necessary for the proof of Theorem 1.1, see [1].

Let $\boldsymbol{C}[[t]]$ be the ring of formal power series in $t$ with coefficients in $\boldsymbol{C}$. We say that the formal power series $\hat{f}(t)=\sum_{n=0}^{\infty} f_{n} t^{n} \in \boldsymbol{C}[[t]]$ has Gevrey order $k>0$ if there exists $C>0$ such that, for all $n \geq 0$

$$
\left|f_{n}\right| \leq C^{n+1} \Gamma\left(1+\frac{n}{k}\right)
$$

where $\Gamma(x)$ is a Gamma function. We denote by $C[[t]]_{1 / k}$ the set of all formal power series with Gevrey order $k$.

A sector on the Riemann surface of the logarithm is the set of the form

$$
S \equiv S(d, \alpha, \rho):=\left\{r e^{i \theta}|| \theta-d \mid<\frac{\alpha}{2}, 0<r<\rho\right\}
$$

where $d$ is an arbitrary real number, $\alpha$ is a positive real and $\rho$ is positive real or $\infty$. In case $\rho=\infty$, we mostly write $S(d, \alpha)$ :

$$
S(d, \alpha) \equiv S(d, \alpha, \infty):=\left\{r e^{i \theta}|| \theta-d \mid<\frac{\alpha}{2}, 0<r\right\} .
$$

A closed subsector $\tilde{S}_{1}$ of $S$ is the set of the form

$$
\tilde{S}_{1} \equiv \tilde{S}_{1}\left(d^{\prime}, \alpha^{\prime}, \rho^{\prime}\right):=\left\{r e^{i \theta}|| \theta-d^{\prime} \mid \leq \frac{\alpha^{\prime}}{2}, 0<r \leq \rho^{\prime}\right\}
$$

where $0<\alpha^{\prime}<\alpha, 0<\rho^{\prime}<\rho,\left|d-d^{\prime}\right|<\alpha / 2-\alpha^{\prime} / 2$.

Given $k>0$, we say that a function $f$ being holomorphic in a sector $S$ asymptotically equals $\hat{f}(t) \in C[[t]]_{1 / k}$ or $\hat{f}(t)$ is the asymptotic expansion of Gevrey order $k$ of $f$ if, for every closed subsector $\tilde{S}_{1}$ of $S$ there exists $C>0$ such that for every $N \geq 0$ and every $t \in \tilde{S}_{1}$

$$
\left|r_{f}(t, N)\right| \leq C^{N+1} \Gamma\left(1+\frac{N}{k}\right),
$$


where $r_{f}(t, N)=t^{-N}\left(f(t)-\sum_{n=0}^{N-1} f_{n} t^{n}\right)$. In such a case, we write, for short, $f(t) \cong_{k} \hat{f}(t)$. We denote by $A_{k}(S)$ the set of all holomorphic functions on $S$ having an asymptotic expansion of Gevrey order $k$.

Definition 2.1. For a formal power series $\hat{f}(t) \in \boldsymbol{C}[[t]]_{1}$ without constant term, the formal Borel transform $\hat{\boldsymbol{B}}_{1} \hat{f}$ is defined by

$$
\hat{B}_{1}: \hat{f}(t)=\sum_{n=1}^{\infty} f_{n} t^{n} \mapsto f(\xi)=\sum_{n=1}^{\infty} f_{n} \frac{\tau^{n-1}}{\Gamma(n)} .
$$

Definition 2.2. For a $\hat{f}(t) \in \boldsymbol{C}[[t]]_{1}$, we say that $\hat{f}(t)$ is 1 -summable in the $d$-direction if there exists an $\varepsilon>0$ such that $\hat{B}_{1} \hat{f} \in A_{1}(S(d, \varepsilon))$ with exponential size at most 1 . We denote by $C\{t\}_{1, d}$ the set of all formal power series that are 1 -summable in the $d$-direction. We say that a function $f(\tau)$ has exponential size at most 1 on $S(d, \varepsilon)$ if there exist $C>0$ and $h>0$ such that

$$
|f(\tau)| \leq C e^{h|\tau|}
$$

on every closed subsector of $S(d, \varepsilon)$.

\section{Estimate of formal solution}

In this section, we show the Gevrey estimate of formal solution.

Proposition 3.1. Assume that $a(x)$ is of exponential size at most 1 . Then, for any $m \in N^{*}$ we have

$$
u_{m}(x)=\frac{(m-1) !}{m}\left(\sum_{j=0}^{m-1}\left(\begin{array}{c}
m-1 \\
j
\end{array}\right) \partial_{x}^{\alpha j} a(x)\right) .
$$

Moreover, we have the estimate: there exist $K>0$ and $h>0$ such that, for any $m \in N^{*}$ and $x \in C$

$$
\left|u_{m}(x)\right| \leq \frac{(m-1) !}{m}\left(1+e^{h \alpha}\right)^{m-1} K e^{h|x|}
$$

Proof. Substituting (1.2) into (1.1) we have

$$
\sum_{j=1}^{\infty} u_{j}(x) j t^{j}=a(x) t+\sum_{j=1}^{\infty} u_{j}(x) j^{2} t^{j+1}+\sum_{j=1}^{\infty} \partial_{x}^{\alpha} u_{j}(x) j^{2} t^{j+1} .
$$

Compare the coefficients of both sides about the power of $t$, to obtain 


$$
\begin{aligned}
u_{1}(x) & =a(x), \\
u_{2}(x) & =\frac{1}{2}\left(a(x)+\partial_{x}^{\alpha} a(x)\right), \\
\vdots & \\
u_{m}(x) & =\frac{(m-1)^{2}}{m}\left(u_{m-1}(x)+\partial_{x}^{\alpha} u_{m-1}(x)\right) \\
& =\frac{(m-1)^{2}}{m}\left(1+\partial_{x}^{\alpha}\right) u_{m-1}(x) .
\end{aligned}
$$

Solving the recurrence relation we have

$$
u_{m}(x)=\frac{(m-1) !}{m}\left(\sum_{j=0}^{m-1}\left(\begin{array}{c}
m-1 \\
j
\end{array}\right) \partial_{x}^{\alpha j} a(x)\right), \quad(m \geq 1)
$$

By assumption, there exist $K>0$ and $h>0$ such that for any $x \in C$

$$
|a(x)| \leq K e^{h|x|} .
$$

Hence, we have

$$
\begin{aligned}
\left|\partial_{x}^{\alpha i} a(x)\right| & \leq \frac{(\alpha i) !}{2 \pi} \int_{\left|x^{\prime}-x\right|=R}\left|\frac{a\left(x^{\prime}\right)}{\left(x^{\prime}-x\right)^{\alpha i+1}}\right|\left|d x^{\prime}\right| \\
& =\frac{(\alpha i) !}{2 \pi} \int_{\left|x^{\prime}-x\right|=R} \frac{K e^{h\left|x^{\prime}\right|}}{R^{\alpha i+1}}\left|d x^{\prime}\right| \\
& =\frac{(\alpha i) !}{R^{\alpha i}} K e^{h(|x|+R)} .
\end{aligned}
$$

By setting $R=\alpha i$ we see that the right-hand side is estimated by $K e^{h(|x|+\alpha i)}$. By (3.1), we have

$$
\begin{aligned}
\left|u_{m}(x)\right| & \leq \frac{(m-1) !}{m}\left(\sum_{j=0}^{m-1}\left(\begin{array}{c}
m-1 \\
j
\end{array}\right)\left|\partial_{x}^{\alpha j} a(x)\right|\right) \\
& \leq \frac{(m-1) !}{m}\left(\sum_{j=0}^{m-1}\left(\begin{array}{c}
m-1 \\
j
\end{array}\right) K e^{h \alpha j} e^{h|x|}\right) \\
& \leq \frac{(m-1) !}{m}\left(1+e^{h \alpha}\right)^{m-1} K e^{h|x|} .
\end{aligned}
$$




\section{Proof of Theorem 1.1}

Set $\partial u / \partial t=: v$. Then, (1.1) is written as follows

$$
t v=a(x) t+t^{2} \frac{\partial}{\partial t} t v+t^{2} \frac{\partial}{\partial t} t \partial_{x}^{\alpha} v
$$

Applying the formal Borel transform to both sides and by setting $\hat{B}_{1}(t v)=\hat{w}$ we have

$$
\hat{w}=a(x)+\tau \hat{w}+\tau \partial_{x}^{\alpha} \hat{w}
$$

where we used

$$
\begin{aligned}
\hat{B}_{1}\left(t^{2} \frac{\partial}{\partial t} t v\right) & =\hat{B}_{1}\left\{\sum_{j=1}^{\infty} u_{j}(x) j^{2} t^{j+1}\right\} \\
& =\sum_{j=1}^{\infty} u_{j}(x) \frac{j}{(j-1) !} \tau^{j}=\tau \hat{B}_{1}(t v) .
\end{aligned}
$$

If we set

$$
\hat{w}=\sum_{n=1}^{\infty} \hat{w}_{n}(\tau) e^{h_{n} x}
$$

then we have

$$
\partial_{x}^{\alpha} \hat{w}=\sum_{n=1}^{\infty} \hat{w}_{n}(\tau) h_{n}^{\alpha} e^{h_{n} x}
$$

Substituting $\hat{w}$ and (1.4) into (4.1) we have

$$
\hat{w}_{n}(\tau)=\frac{C_{n}}{1-\tau\left(1+h_{n}^{\alpha}\right)}
$$

for every $n \in N^{*}$.

Take $R \in(0,1)$ and let $|\tau|<R$. Choose $N \in N^{*}$ such that $\left|1 /\left(h_{n}^{\alpha}+1\right)\right| \geq R$ for every $n \geq N+1$. We consider

$$
\sum_{n=1}^{\infty} \hat{w}_{n}(\tau) e^{h_{n} x}=\sum_{n=1}^{N} \hat{w}_{n}(\tau) e^{h_{n} x}+\sum_{n=N+1}^{\infty} \hat{w}_{n}(\tau) e^{h_{n} x}
$$


Singular points of the first term in the right-hand side are given by $\{\tau=$ $\left.1 /\left(h_{n}^{\alpha}+1\right) \mid n=1, \ldots, N\right\}$. According to the assumption on $h_{n}^{\alpha}$, we have

$$
R \leq\left|1+h_{N+1}^{\alpha}\right|^{-1} \leq\left|1+h_{N+2}^{\alpha}\right|^{-1} \leq \cdots<1 .
$$

So, there exists $A_{0} \in \boldsymbol{R}$ such that $\sup _{n \geq N+1,|\tau|<R}\left\{1 /\left(1-|\tau|\left|1+h_{n}^{\alpha}\right|\right)\right\}=A_{0}$. It follows that

$$
\begin{aligned}
\left|\sum_{n=N+1}^{\infty} \frac{C_{n}}{1-\tau\left(1+h_{n}^{\alpha}\right)} e^{h_{n} x}\right| & \leq \sum_{n=N+1}^{\infty} \frac{1}{1-|\tau|\left|1+h_{n}^{\alpha}\right|}\left|C_{n}\right|\left|e^{h_{n} x}\right| \\
& \leq A_{0} \exp \left(3^{\alpha^{-1}}|x|\right) \sum_{n=N+1}^{\infty}\left|C_{n}\right|<\infty
\end{aligned}
$$

for $|\tau|<R$. Since $R$ is arbitrary, $\sum_{n=1}^{\infty} \hat{w}_{n}(\tau) e^{h_{n} x}$ is a meromorphic function on $|\tau|<1$.

By Lemma 1.1, we have (1.5) and (1.6) for $v=n-q(q-1) / 2$ and $q(q-1) / 2<n \leq q(q+1) / 2$. It follows that $1 \leq v \leq q$. We note that $q$ tends to infinity as $n \rightarrow \infty$. Hence, the set $\left\{1 /\left(1+h_{n}^{\alpha}\right)\right\}_{n}$ accumulates to any points on the unit circle. Therefore, $v$ is not 1 -summable in any direction.

\section{Acknowledgement}

The author wishes to thank Prof. Yamazawa for constructive discussions.

\section{References}

[1] Balser, W., Formal power series and linear systems of meromorphic ordinary differential equations, Springer-Verlag, New York, 2000.

[2] Hibino, M., Borel summability of divergent solutions for singular first order linear partial differential equations with polynomial coefficients, J. Math. Sci. Univ. Tokyo, 10 (2003), 279-309.

[ 3 ] Lutz, D. A., Miyake, M. and Schäfke, R., On the Borel summability of divergent solutions of the heat equation, Nagoya Math. J., 154 (1999), 1-29.

[4] Malek, S., On the summability of formal solutions of linear partial differential equations, J. Dyn. Control Syst., 11 (2005), 389-403.

[5] Ōuchi, S., Genuine solutions and formal solutions with Gevrey type estimates of nonlinear partial differential equations, J. Math. Sci. Univ. Tokyo, 2 (1995), 375-417.

[6] Ōuchi, S., Multisummability of formal solutions of some linear partial differential equations, J. Differential Equations, 185 (2002), 513-549.

[7] Ōuchi, S., Multisummability of formal power series solutions of nonlinear partial differential equations in complex domains, Asymptot. Anal., 47 (2006), 187-225.

[8] Tahara, H. and Yamazawa, H., Multisummability of formal solutions to the Cauchy problem for some linear partial differential equations, J. Differential Equations, 255 (2013), 3592-3637. 


\author{
nuna adreso: \\ Department of Mathematics \\ Graduate School of Science \\ Hiroshima University \\ 1-3-1 Kagamiyama \\ Higashi-Hiroshima, 739-8526 \\ Japan
}

(Recevita la 30-an de aprilo, 2016)

(Revizita la 21-an de septembro, 2016) 